\title{
editorial
}

\section{Indicaciones y resultados del trasplante de páncreas}

\begin{abstract}
6
G1 primer trasplante experimental de páncreas, realizado por Hedon ${ }^{1}$, se remonta al año 1892, colocando una porción de páncreas en el tejido subcutáneo, previniendo así el desarrollo de diabetes en el perro. El descubrimiento de la insulina por Banting y Best ${ }^{2}$, en 1922, frenó la investigación en trasplante de páncreas, ya que el tratamiento de los diabéticos con esta hormona alargaba la supervivencia, objetivándose, sin embargo, las complicaciones evolutivas de esta enfermedad, anteriormente no observadas por el fallecimiento precoz de estos enfermos, y que a partir de dicho momento constituyeron las causas principales de morbi-mortalidad. Así, a finales de los sesenta y principios de los setenta, se empezaron a tratar estas complicaciones, tales como la nefropatía terminal con trasplante renal y la retinopatía, con láser. A pesar de estos tratamientos, la diabetes y sus complicaciones evolutivas persistían, continuándose los trabajos experimentales hasta realizarse el primer trasplante en humanos, en 1966, por Kelly et al. ${ }^{3}$, en la Universidad de Minnesota. El trasplante fue pancreático-renal simultáneo, recuperando el enfermo el estado euglucémico, pero falleciendo a los dos meses por rechazo y sepsis. Posteriormente, Lillehei et al. ${ }^{4}$, también en Minnesota, trasplantaron una serie de 13 pacientes, consiguiendo que sólo un injerto funcionara al cabo de un año. Otros cirujanos, como Gliedman, en Nueva York, Groth, en Estocolmo, y Sutherland, en Minnesota, continuaron esta experiencia, de manera que hasta agosto del 2000 se habían registrado 13.917 trasplantes pancreáticos en todo el mundo ${ }^{5}$. Hoy en día, el trasplante de páncreas es una técnica aceptada de forma generalizada y el único tratamiento conocido con el que se consigue un estado euglucémico insulino-independiente con normalización de la hemoglobina glicosilada, mejoría de la calidad de vida y de las complicaciones de la diabetes ${ }^{6}$.
\end{abstract}

\section{INDICACIONES Y CONTRAINDICACIONES DEL TRASPLANTE PANCREÁTICO}

Los criterios de inclusión y exclusión para los candidatos a trasplante pancreático varían poco entre los distintos grupos de trasplante ${ }^{7-11}$.

El momento idóneo para realizar un trasplante de páncreas sería antes de la aparición de las complicaciones de la diabetes. No obstante, debido a lo difícil que resulta predecir cuándo aparecerán estas complicaciones, la mayoría de los trasplantes se llevan a cabo en una fase evolutiva avanzada, es decir, en presencia de una triopatía (nefropatía, neuropatía 
y retinopatía). A tenor de que estas complicaciones son irreversibles, se ha ensayado el trasplante pancreático en la fase más precoz (prediálisis), bien realizándolo aislado (aclaramiento de creatinina $>70 \mathrm{ml} / \mathrm{min}$ ) o bien combinándolo con el renal (aclaramiento $<40 \mathrm{ml} / \mathrm{min}$ ), llegando así a conseguir unos resultados similares a los obtenidos en enfermos dializados en cuanto a la supervivencia del injerto y del enfermo, pero con la ventaja adicional de conseguir una rehabilitación mejor y más precoz en los no dializados ${ }^{10}$. Cuando el aclaramiento se halla entre 40-60 ml/min se trata con CyA o FK 506 hasta alcanzar niveles terapéuticos y ver la repercusión sobre éste: si es $>60 \mathrm{ml} / \mathrm{min}$, se debe realizar un trasplante pancreático aislado y si es $<60$, la indicación es de un trasplante pancreático-renal.

La relación de criterios de exclusión sigue casi paralela a la de otros trasplantes de órganos, estableciendo una serie de contraindicaciones absolutas (gangrena periférica progresiva, infecciones activas, enfermedad neuro-psiquiátrica grave, enfermedad tumoral, salvo cirugía 5 años antes y sin recidiva) y otra de relativas (edad mayor de 60 años, ateroma generalizado de arterias ilíacas, neuropatía periférica incapacitante, o cualquier tipo de insuficiencia terminal: cardiaca, respiratoria o hepática, salvo que se realice antes el trasplante de estos órganos).

Además, algunos autores ${ }^{12}$ contraindican el trasplante en los enfermos con ceguera, cardiopatía severa o amputaciones mayores. Asimismo, otros grupos ${ }^{13}$ con gran experiencia son aún más estrictos en cuanto a las contraindicaciones, al no relizar el trasplante cuando el enfermo es mayor de 60 años, es fumador activo, la fracción de eyección ventricular izquierda es menor del $40 \%$ y presenta obesidad severa (mayor del $50 \%$ del peso ideal). Hasta hace poco, el trasplante en la diabetes tipo 2 constituía una contraindicación; sin embargo, algunos equipos ${ }^{14,15}$ han realizado el trasplante pancreático-renal simultáneo en un grupo reducido de dichos enfermos obteniendo resultados similares a los conseguidos en la diabetes tipo 1 .

\section{EVALUACIÓN PREOPERATORIA DE LOS RECEPTORES}

Los protocolos de evaluación preoperatoria de los candidatos presentan escasas variaciones entre los diferentes grupos de trasplante, ${ }^{7,10}$. Resumiendo, debe realizarse una historia clínica detallada, valoración oftalmológica (retinopatía), neurológica (neuropatía periférica), vascular periférica (pulsos de MMII, TAC helicoidal aorto-ilíaco para evaluar placas de ateroma), urológica (cistouretrografía y cistomanometría cuando se hace derivación pancreato-duodeno-vesical), digestiva (vaciamiento gástrico isotópico para evaluar la neuropatía vegetativa).

Va a ser fundamental una valoración cardiológica: Rx de tórax, ECG y prueba de esfuerzo con talio. La coronariografía se realiza en caso de sos- 
pecha de cardiopatía: edad mayor de 45 años, prueba de esfuerzo patológica, diabetes de más de 25 años de evolución, fumador importante, HTA de larga duración, enfermedad cerebrovascular o vascular periférica ${ }^{16}$. No obstante, una historia de infarto miocárdico, angioplastia o bypass arterial coronario, no constituyen contraindicaciones absolutas para realizar el trasplante pancreático, si mantienen una reserva funcional aceptable ${ }^{17}$.

La función endocrina pancreática se estudia mediante la determinación basal y post-estímulo de la glucemia y el péptido $\mathrm{C}$, hemoglobina glicosilada, Ac anti-islotes y Ac anti-insulina, mientras que la función exocrina se evalúa mediante la determinación de los niveles de amilasa y lipasa en sangre y orina. El protocolo pre-trasplante se completa con la realización de análisis sistemáticos de sangre y orina, serología viral, cultivos, estudio de histocompatibilidad, etc.

\section{TIPOS DE TRASPLANTE}

1. Trasplante pancreático-renal simultáneo. Existe un consenso general en cuanto a que el trasplante de riñón es el mejor tratamiento de la nefropatía diabética avanzada ${ }^{18}$. La asociación de un trasplante pancreático al renal ha sido motivo de controversia por el eventual riesgo de incrementar la tasa de morbi-mortalidad. Sin embargo, se ha referido un aumento de la supervivencia del riñón cuando se efectúa un trasplante pancreático-renal simultáneo en comparación a cuando se hace un renal aislado en el seno de una nefropatía diabética terminal ${ }^{19,20}$. De este modo, el trasplante pancreático-renal simultáneo ha sido rápidamente aceptado como la mejor opción terapéutica para los pacientes con nefropatía diabética terminal avanzada, ya que el doble trasplante elimina la necesidad de la diálisis y la administración exógena de insulina, obteniendo así un buen control metabólico y una apreciable mejoría de la calidad de vida $^{13}$ previniendo además la recidiva de la nefropatía en los diabéticos tipo 1 trasplantados solamente de riñón, hecho que sucede en la mitad de estos receptores ${ }^{21}$.

2. Trasplante pancreático después del renal. En diabéticos con trasplante renal normofuncionante y con pocos o ningún episodio de rechazo renal, el trasplante pancreático adicional y posterior (procedente de otro donante) ha sido defendido en base a que el receptor ya está obligado, de por vida, a una inmunosupresión que no cambiará con el nuevo injerto $^{22}$, no suponiendo el nuevo trasplante otro riesgo para el receptor que el inherente al acto quirúrgico. Otro argumento para añadir el trasplante de páncreas al renal previo es la presencia de una glucemia inestable con requerimientos variables de insulina y tendencia al desarrollo de las complicaciones de la diabetes.

3. Trasplante pancreático aislado. Este debería realizarse, de forma ideal, antes de la aparición de las complicaciones asociadas a la diabe- 
tes, tales como la nefropatía que, una vez avanzada, sería subsidiaria de trasplante renal. Por otro lado, en el momento actual, no disponemos de marcadores que nos hagan predecir qué pacientes van a desarrollar complicaciones progresivas y graves ${ }^{23}$. Así pues, el trasplante pancreático aislado quedaría restringido para los diabéticos tipo 1 sin insuficiencia renal pero de difícil tratamiento insulínico por su hiperlabilidad manifiesta ${ }^{1}$ y para los que tienden al desarrollo de complicaciones diabéticas precoces (retinopatía, nefropatía, neuropatía periférica, neuropatía autonómica, etc.) que, al fin, serían más graves que las eventuales asociadas a la inmunosupresión crónica ${ }^{15}$. Con la aparición de nuevos inmunosupresores, más selectivos y con menos efectos secundarios, se puede ampliar en el futuro el abanico de indicaciones.

\section{SELECCIÓN DE DONANTES DE PÁNCREAS}

En principio, casi todos los cadáveres con muerte cerebral son apropiados para la extracción multiorgánica: riñones, corazón y pulmones, añadiendo la del páncreas, a menos que los potenciales donantes sean diabéticos o presenten un traumatismo pancreático ${ }^{24}$. En general, se aceptan los injertos pancreáticos de donantes entre 3-55 años con un límite extremo inferior de $18 \mathrm{~kg}$ de peso, sin antecedentes de diabetes tipo 1 ó 2 del donante o diabetes tipo 1 en familiares de primer grado y ausencia de pancreatitis, calcificaciones, traumatismo pancreático, esplenectomía, contaminación bacteriana abdominal, tumor (excepto piel o cerebro), infección y drogadicción i.v., debiendo ser normal el color y la consistencia del páncreas ${ }^{24-26}$. La presencia de hiperglucemia, hiperamilasemia o antecedentes de alcoholismo se consideran contraindicaciones relativas.

\section{TÉCNICA DEL TRASPLANTE DE PÁNCREAS}

En el donante se extrae el páncreas completo junto con el bazo y la primera, segunda y tercera porciones duodenales, perfundiéndose con una solución de Wisconsin y conservándose a unos $4^{\circ} \mathrm{C}$, rodeado de suero salino y hielo, de manera que así puede permanecer apto para trasplante hasta 24 horas, aunque lo habitual es implantarlo en un plazo de 9-12 horas de isquemia fría. El injerto que se implantará en el receptor corresponde al páncreas completo con los $5-7 \mathrm{~cm}$ del marco duodenal que lo rodea. La vena porta del injerto se anastomosa con la vena ilíaca común o externa del receptor y la arteria mesentérica superior (AMS) y arteria esplénica del injerto se unen mediante la interposición de un injerto en Y invertida formado por la arteria ilíaca común (rama vertical de la Y que se anastomosa con la arteria ilíaca común 
del receptor), la interna (anastomosis con la arteria esplénica del donante) y la externa (anastomosis con la AMS del donante). El drenaje de la secreción exocrina del injerto pancreático se puede realizar mediante una derivación duodeno-vesical, teniendo la ventaja de poder medir la actividad de la amilasa en orina (AAO), excelente marcador del rechazo del injerto, pero asociada a patología urinaria (infecciones, hematuria, fístulas) y acidosis metabólica, o bien mediante una duodeno-yeyunostomía, técnica más fisiológica que la anterior pero de mayor gravedad cuando se presentan fístulas anastomóticas por las infecciones intraabdominales que provoca. Después de la implantación del páncreas en fosa ilíaca derecha se implanta el riñón en la izquierda.

\section{MONITORIZACIÓN E INMUNOSUPRESIÓN DEL INJERTO PANCREÁTICO}

Una vez finalizado el trasplante pancreático-renal, incluso durante el primer día, si el páncreas funciona normal, el enfermo puede hallarse euglucémico sin necesidad de insulina o como mucho precisar pequeñas dosis cuando la glucemia es mayor de $150 \mathrm{mg} / \mathrm{dl}$.

Los principios que rigen la inmunosupresión en el trasplante de páncreas son similares a los de otros órganos sólidos. La supervivencia del trasplante pancreático-renal simultáneo es mayor que cuando se realiza después del renal (diferentes donantes) o de forma aislada, siendo debido a los efectos inmunosupresor de la uremia y protector del riñón sobre el rechazo pancreático, ya que, en la fase precoz de éste, la creatinina aparece elevada como única manifestación en el $50 \%$ de los casos y asociada a un descenso del $50 \%$ de la AAO en un $40 \%$ de los casos, mientras que un descenso aislado de la AAO sólo se presenta en el 10\%. Estas son las razones por las cuales el trasplante pancreático-renal tiene una menor tasa de rechazo ${ }^{27}$.

La introducción de nuevos inmunosupresores, tales como el FK 506 y Micofenolato Mofetil (MMF) ha supuesto una reducción significativa de las tasas de rechazo (11-25\% en pancreático-renal y $52 \%$ en pancreático aislado) con respecto a la utilización previa de CyA y azatioprina como base de la inmunosupresión (61 y $96 \%$, respectivamente) ${ }^{28,29}$.

El regimen inmunosupresor más utilizado en la actualidad incluye: inducción durante 4-8 días con anticuerpos antilinfocitarios (ATG u OKT3), corticoides, MMF y FK 506. La rapamicina es un inmunosupresor de reciente introducción en el trasplante de islotes y de páncreas completo, que permite reducir las dosis de FK 506 y así prevenir los efectos secundarios de éste a dosis altas (diabetogenicidad, nefrotoxicidad). El rechazo agudo se trata con 250-500 mg/día de metilprednisolona, durante 3 días, añadiendo ATG u OKT3 durante 7-14 días si el rechazo es córtico-resistente ${ }^{30}$. 


\section{COMPLICACIONES POSTRASPLANTE}

Las complicaciones quirúrgicas más frecuentes son las pancreatitis, abscesos intrapancreáticos, colecciones peripancreáticas, pseudoquistes, fístulas pancreáticas y anastomóticas y complicaciones vasculares (trombosis, hemorragia, pseudoaneurismas y fístulas arteriovenosas).

La trombosis del injerto se presenta en el $12 \%$ de los casos (venosa en el 7\% y arterial en el 5\%), siendo la causa de la pérdida del páncreas en el 10-20\% de los casos, atribuyéndose a la situación de bajo flujo y estancamiento de la sangre por tener el páncreas una vascularización arterial de grandes vasos y un drenaje escaso procedente de las ramas pancreáticas ${ }^{31}$. Para algunos autores la heparinización reduce el riesgo de trombosis ${ }^{32}$, mientras que para otros ${ }^{33}$ no cambia la incidencia de esta complicación casi siempre seguida de la pérdida del injerto.

\section{SUPERVIVENCIA}

Según el Registro Internacional de Trasplante de Páncreas ${ }^{5}$, de los 4.208 trasplantes pancreático-renales realizados en EE.UU., entre los años 1996-2000, el 95\% de los enfermos sobrevivían al año del trasplante, correspondiendo al 94\% la supervivencia de los 575 enfermos trasplantados de páncreas después del riñon y al 98\% la supervivencia de los 255 enfermos trasplantados solamente de páncreas. La supervivencia del injerto pancreático al año era del 84,73 y $70 \%$, según que se hiciera un trasplante pancreático renal simultáneo, un pancreático después del renal o un pancreático aislado, respectivamente, mientras que la del riñón durante el mismo periodo era del $88 \%{ }^{5}$. Si tenemos en cuenta los 212 trasplantes pancreático-renales simultáneos realizados por el equipo de la Universidad de Minnesota, entre los años 1994-2000, la supervivencia del enfermo, del páncreas y del riñón, a los 5 años, era del 88,73 y $81 \%$, respectivamente ${ }^{15}$. Las causas más frecuentes de pérdida del injerto pancreático son: trombosis, rechazo, fístulas, infecciones, hemorragia, etc. ${ }^{34}$.

\section{BENEFICIOS DEL TRASPLANTE DE PÁNCREAS}

Las consecuencias inmediatas de un trasplante pancreático funcionante son la normalización de la glucemia y la hemoglobina glicosilada, que se mantiene mientras funciona el injerto, y la aparición de una hiperinsulinemia sobre todo cuando se hace una anastomosis venosa porto-sistémica ${ }^{35}$. A pesar de este hiperinsulinismo, las pruebas del metabolismo de los carbohidratos permanecen normales ${ }^{36}$. Otro 
efecto beneficioso de un trasplante pancreático-renal normofuncionante es la normalización del colesterol y triglicéridos en ayunas, aumentando el HDL y descendiendo la lipemia postprandial, hallazgos predictivos de un bajo riesgo de enfermedad cardiovascular en la población general ${ }^{37}$.

La mayoría de los candidatos a trasplante presentan una retinopatía proliferativa que se suele estabilizar o mejorar después de 3-4 años de un trasplante pancreático con funcionamiento normal ${ }^{15,38}$.

Un estado euglucémico de 5-10 años de duración después de un trasplante pancreático aislado puede hacer revertir una nefropatía leve-moderada previamente evidenciada antes del trasplante ${ }^{39}$. Los enfermos con nefropatía diabética terminal trasplantados de páncreas-riñón mantienen una función renal estable, mientras que los trasplantados sólo de riñón presentan un deterioro funcional de éste a los dos años de seguimiento ${ }^{40}$.

La neuropatía, sintomática en el $80 \%$ de los receptores, puede hacerse reversible con un mejor control de la glucemia (trasplante de páncreas) y después de un trasplante renal aislado por el hecho de la desaparición de la uremia ${ }^{41}$.

\section{CALIDAD DE VIDA}

Si antes del trasplante sólo el 55\% desarrollaba una actividad social normal, después aumenta hasta un $83 \%{ }^{10}$. Esta calidad de vida se ve acrecentada en los diabéticos que reciben un trasplante pancreático-renal en vez de un trasplante renal aislado ${ }^{20,42}$. Los diabéticos lábiles, con gran dificultad para su control metabólico o que presentan hipoglucemias inesperadas, mejoran ostensiblemente su calidad de vida después de hacerse insulino-independientes por el trasplante pancreático $^{42}$. Como prueba de calidad de vida esperada o conseguida, casi el $100 \%$ de los enfermos con injertos funcionantes y el $85 \%$ de los que les han fracasado aconsejan a otros enfermos el trasplante de páncreas ${ }^{43}$. Por último, es interesante reseñar que los enfermos que han perdido uno de los injertos, renal o pancreático, sugieren que la insulino-independencia mejora más la calidad de vida que la independencia de la diálisis ${ }^{15}$.

C. Jiménez, E. Moreno, A. Andrés* Servicio de Cirugía General A parato Digestivo y TrasplantedeÓrganos A bdominales. *Servicio de Nefrología. H ospital Doce de Octubre M adrid. 


\section{BIBLIOGRAFÍA}

1. Hedon E. Greffe sous-cutanée du pancréas. Archs Physiol Norm Pathol 1892; 5: 617-28.

2. Banting FG, Best CR. The internal secretion of the pancreas. J Lat Clin Med 1922; 7: 251-66.

3. Kelly WD, Lillehei RC, Merkel FK, Idezuki Y, Goetz FC. Allotransplantation of the pancreas and duodenum along with the kidney in diabetic nephropathy. Surgery 1967; 61: 827-37.

4. Lillehei RC, Ruiz JO, Acquino C, Goetz F. Transplantation of the pancreas. Acta Endocrinol 1976; 83: 303- 18.

5. International Pancreas Transplant Registry. Year-end update October 2000. University of Minnesota. Department of Surgery. Gruessner RWG, Sutherland DER.

6. Robertson RP. Pancreatic and islet transplantation diabetes. N Engl J Med 1992; 327: 1861-8.

7. The University of Michigan Pancreas Transplant Evaluation Committee. Pancreatic transplantation as treatment for IDDM. Diabetes Care 1988; 11: 669-75.

8. Velosa JA, Frohnert PP, Perkins JD, Zimmerman BR, Fromme GA, Geerdes RB. Pancreas transplantation at Mayo: I. Patient Selection. Mayo Clin Proc 1990; 65:4 75-82.

9. Sollinger HW, Knechtle SJ, Reed A, D'Alessandro, Kalayoglu M, Belzer FO, Pirsch J. Experience with 100 consecutive simultaneous kidney-pancreas transplants with bladder drainage. Ann Surg 1991; 214: 703- 11.

10. Stratta RJ, Taylor RJ, Bynon JS. Surgical treatment of diabetes mellitus with pancreas transplantation. Ann Surg 1994; 220: 809-17.

11. American Diabetes Association. Pancreas transplantation for patients with type 1 diabetes. Diabetes Care 2000; 23: S85.

12. Sollinger HW, Ploeg RJ, Eckhoff DE, Stegall MD, Isaacs R, Pirsch JD, et al. Two hundred consecutive simultaneous pancreas-kidney transplants with bladder drainage. Surgery 1993; 114: 736- 44.

13. Stratta Rj, Taylor RJ, Gill IS. Pancreas transplantation: a managed cure approach to diabetes. Curr Probl Surg 1996; 33:712-808.

14. Sasaki TM, Gray RS, Ratner RE, Currier C, Aquino A, Barhyte DY, et al. Sucessful long-term kidney-pancreas in diabetic patients with high C-peptide levels. Transplantation 1999; 67: 586A.

15. Sutherland DER, Gruessner RWG, Dunn DL, Matas AJ, Humar A, Kandaswamy R, et al. Lessons from more than 1,000 pancreas transplants at a single institution. Ann Surg 2001; 233: 463-501.

16. Morrow CE, Schwartz JS, Sutherland DER, Simmons RL, Ferguson RM, Kjellstrand CM, et al. Predictive value of thallium stress testing for coronary and cardiovascular events in uremic diabetic patients before renal transplantation. Am J Surg 1983; 146: 331-5.

17. Manske $\mathrm{CL}$, Thomas W, Wang $\mathrm{Y}$, Wilson RF. Screening diabetic transplant candidates for coronary artery disease: identification of a low risk subgroup. Kidney Int 1993; 44: 617-21.

18. Najarian JS, Kaufman DB, Fryd DS, M cHugh L, Mauer SM, Ramsay RC, et al. Long-term survival following kidney transplantation in 100 type 1 diabetic patients. Transplantation 1989; 47: 106-13.

19. Cheung AHS, Sutherland DER, Gillinghan KJ, McHugh LE, Moudry- Munns $\mathrm{KC}$, Dunn DL, et al. Simultaneous pancreas-kidney transplant alone in diabetic patients. Kidney Int 1992; 41: 924-9.

20. Stratta RJ, Taylor RJ, Ozaki CF, Bynon JS, Miller SA, Baker TL, et al. The analysis of benefit and risk of combined pancreatic and renal transplantation versus renal transplantation alone. Surg Gynecol Obstet 1993; 177: 163-71. 
21. Mauer SM, Goetz FC, McHugh LE, Sutherland DER, Barbosa J, Najarian JS, et al. Long-term study of normal kidneys into patients with type I diabetes. Diabetes 1989; 38: 516-23.

22. Sutherland DER. Indications for pancreas transplantation: a commentary. Clin Transplant 1990; 4: 242-6.

23. Nathan DM. Long-term complications of diabetes mellitus. N Engl J Med 1993; 328: 1676-84.

24. Sutherland DER, Goetz C, Najarian JS. Pancreas transplantation at the University of Minnesota: donor and recipient selection, operative and postoperative management, and outcome. Transplant Proc 1987; 19: 63- 74

25. Groth CG, Lundgren G, Wilczek $\mathrm{H}$. The pancreatic graft and its retrieval from the cadaveric donor. En: Groth CG, ed. Pancreatic Transplantation. Philadelphia: WB Saunders Co., 1988. p. 49-60.

26. Perkins JD, Frohnert PP, Service FJ, Wilhelm MP, Keating MR, DiCecco SR, et al. Pancreas transplantation at Mayo: III: Multidisciplinary management. Mayo Clin Proc 1990; 65: 496- 508.

27. Sutherland DER. Inmunosuppression for clinical pancreas transplantation. Clin Transplan 1991; 5: 549-53.

28. Gruessner RWG, Dunn DL, Tzardis PJ, Tomadze G, M oudry-Munns KC, Matas AJ, et al. Simultaneous pancreas and kidney transplants versus single kidney transplants and previous kidney transplants in uremic patients and single pancreas transplants in non- uremia diabetic patients: comparison of rejection, morbidity and long-term outcome. Transplant Proc 1990; 22: 622-3.

29. Gruessner RWG, Sutherland DER, Drangstveit MB, West M, Gruessner A. Micophenolate mofetil and tacrolimus for induction and maintenance therapy after pancreas transplantation. Transplant Proc 1998; 30: 518-20.

30. Odorico JS, Pirsch JD, Knechtle SJ, D 'Alessandro AM, Sollinger HW. A study comparing micophenolate mofetil to azathioprine in simultaneous pancreas- kidney transplantation. Transplantation 1998; 66: 1751-9.

31. Groth CG. Surgical complications following pancreatic transplantation. En: Carl G Groth, ed. Pancreatic transplantation. Philadelphia: WB Sanders Company, 1988. p. 219-38.

32. Humar A, Kandaswamy R, Granger D, Gruessner RW, Gruessner A, Sutherland DER. Decreased surgical risks of pancreas transplantation in the modern era. Ann Surg 2000; 231: 269-75.

33. Sollinger HW. Pancreatic transplantation and vascular graft thrombosis. J Am Coll Surg 1996; 182: 362-3.

34. Sollinger HW, Odorico JS, Knectle SJ, D’Alessandro AM, Kalayoglu M, Pirsch JD. Experience with 500 simultaneous pancreas-kidney transplants. Ann Surg 1998; 228: 284-96.

35. Robertson RP, Abid M, Sutherland DER, Diem D. Glucose homeostasis and insulin secretion in human recipients of pancreas transplantation. Diabetes 1989; 38(Supl): 97-8.

36. Katz H, Homan M, Velosa J, Robertson P, Rizza R. Effects of pancreas transplantation on postprandial glucose metabolism. N Engl J Med 1991; 325: 1278-83.

37. Foger B, Konigsrainer A, Palos G, Brandstätter E, Ritsch A, König P, et al. Effect of pancreas transplantation on lipoprotein lipase, postprandial lipemia, and HDL cholesterol. Transplantation 1994; 58: 899-904.

38. Wang Q, Klein R, Moss SE, Klein BEK, Hoyer C, Burke K, et al. The influence of combined kidney-pancreas transplantation on the progression of retinopathy: a cases series. Ophtalmology 1994; 101: 1071-6.

39. Fioretto $P$, Steffes MW, Sutherland DER, Goetz FC, Mauer M. Reversal of lesions of diabetic nephropathy after pancreas transplantation. $\mathrm{N}$ Engl J Med 1998; 339: 69- 75. 
40. El-Gebely S, Hathaway DK, Elmer DS, Gaber LW, Acchiardo S, Gaber AO. An analysis of renal function in pancreas-kidney and diabetic kidney alone recipients at two years following transplantation. Transplantation 1995; 59: 1410-5.

41. The Diabetes Control Group and Complications Trial Research Group. The effect of intensive treatment of diabetes on the development and progression of long-term complications in insulin-dependent diabetes mellitus. N Engl J Med 1993; 29: 977-86.

42. Gross CR, Zehrer CL. Health-related qualify of life in pancreas transplant recipients. Clin Transplant 1992; 6: 165-71.

43. Zehrer CL, Gross CR. Quality of life of pancreas transplant recipients. Diabetología 1991; 34 (Supl 1): S145-S149. 